\title{
Variabilité hydrologique et biologique du golfe du Lion. I. Transports en azote et productivité potentielle
}

\author{
Pascal CONAN $^{a *}$, Mireille PUJO-PAY ${ }^{b}$, Patrick RAIMBAULT ${ }^{a}$, Michel LEVEAU ${ }^{a}$ \\ "Centre d'océanologie de Marseille, LOB-UMR 6535, faculté des sciences de Luminy, case 901, 13288 Marseille \\ cedex 09, France \\ * conan@com.univ-mrs.fr \\ ${ }^{\text {b }}$ Laboratoire d'océanologie biologique de Banyuls, UMR 7621, laboratoire Arago, BP 44, 66651 Banyuls-sur-Mer cedex, \\ France
}

(Révisé le 27 octobre 1998, accepté le 28 octobre 1998)

\begin{abstract}
Hydrological and biological variability in the Gulf of Lions. I. Nitrogen fluxes and potential productivity. A monthly time-series of the seasonal evolution of the North Mediterranean Current (NMC) and of nitrogen flux across a transect $\left(5^{\circ} 12^{\prime} 5 \mathrm{E}\right.$ between $43^{\circ} 10^{\prime} \mathrm{N}$ and $\left.42^{\circ} 50^{\prime} \mathrm{N}\right)$ at the entrance of the Gulf of Lions was carried out over two years. The NMC exhibited two distinct structures: a winter structure (from November to May) characterised by strong mesoscale and interannual variations in which the current was 20 to $30 \mathrm{~km}$ width, about $400 \mathrm{~m}$ depth and velocity scaled between 5 and more than $40 \mathrm{~cm} \mathrm{~s}^{-1}$; and a summer structure (from May to November) which was less sensitive to mesoscale variations but showing large interannual variability. The current was located more offshore, was wider and shallower than the one in winter and velocity remained below $20 \mathrm{~cm} \mathrm{~s}^{-1}$. Meteorological conditions, frontal structures on both sides of the NMC, coastal upwelling and continental inputs explained the observed mesoscale discrepancies between water flux and nitrogen transport. Nitrogen input from the general circulation across the prospected transect is estimated at $187 \pm 20 \mathrm{kT} \mathrm{a}^{-1}$ in the $0-200 \mathrm{~m}$ layer, with a distribution of $39 \%$ nitrate, $6 \%$ particulate nitrogen and $55 \%$ dissolved organic nitrogen. Nitrate input $\left(25 \pm 3 \mathrm{kT} \mathrm{a}^{1}\right)$ in the 0-100 $\mathrm{m}$ upper layer could sustain more than one third of the potential new production in the Gulf of Lions. (C) Elsevier, Paris
\end{abstract}

\section{hydrology / mesoscale variability / nitrogen / flux / budget}

Résumé - Un suivi mensuel de l'évolution saisonnière du Courant Nord Méditerranéen (CNM) et des flux en azote à travers une radiale $\left(5^{\circ} 12^{\prime} 5 \mathrm{E}\right.$ entre $43^{\circ} 10^{\prime} \mathrm{N}$ et $\left.42^{\circ} 50^{\prime} \mathrm{N}\right)$ a été réalisé, à l'entrée du golfe du Lion, sur une période de deux ans. Le CNM est décrit selon deux structures distinctes. Une structure hivernale (de novembre à mai) caractérisée par de fortes variations à méso-échelle et interannuelles ; la veine de courant est large de 20 à $30 \mathrm{~km}$ et profonde d'environ $400 \mathrm{~m}$, avec des vitesses comprises entre 5 et plus de $40 \mathrm{~cm} \mathrm{~s}^{-1}$. Une structure estivale (entre mai et novembre) moins sensible aux variations à méso-échelle mais présentant une forte variabilité interannuelle ; la veine est alors plus hauturière, plus large et moins profonde qu'en hiver, les vitesses restant généralement inférieures à $20 \mathrm{~cm} \mathrm{~s}^{-1}$. Les conditions météorologiques, la présence de zones frontales sur les bords interne et externe du CNM, les phénomènes d'upwelling côtier et d'apports continentaux permettent d'expliquer les découplages observés entre les flux en eau et en azote à travers la radiale. Les apports en azote par la circulation générale à travers la radiale sont estimés à $187 \pm 20 \mathrm{kT} \mathrm{a}^{-1}$ dans la couche $0-200 \mathrm{~m}$, et se répartissent en $39 \%$ de nitrate, $6 \%$ d'azote particulaire et $55 \%$ d'azote organique dissous. Les apports de nitrate dans la couche superficielle $0-100 \mathrm{~m}\left(25 \pm 3 \mathrm{kT} \mathrm{a}^{-1}\right)$ pourraient être à l'origine de près d'un tiers de la production nouvelle potentielle du golfe du Lion. (C) Elsevier, Paris

hydrologie / variabilité à méso-échelle / azote / flux de matière / bilan 


\section{INTKODUCTION}

Le golfe du Lion couvre une superficie marine d'environ $11000 \mathrm{~km}^{2}$. Limité au N-E par le cap Croisette et au S-O par le cap Creus, il reçoit d'importants apports continentaux [29] sur un large plateau continental quasi hémisphérique (jusqu'à $70 \mathrm{~km}$ de largeur), creusé de protonds canyons. De nombreuses études sont consacrées au plateau continental et au panache rhodanien $[12,14,29,33$, $34,44]$, ainsi qu'aux régions voisines telles que la mer Ligure [ $1,4,19,39]$ et la mer Catalane [17, 18]. Les données relatives au golfe du Lion, notamment aux zones du large, restent peu abondantes [7, 27, 28, 41].

En Méditerranée nord-occidentale, les masses d'eau décrivent un circuit cyclonique (figure la). En surface, l'eau originaire du flux atlantique, entrant par le détroit de Gilbraltar (MAW, Modified Atlantic Water), s'écoule de part et d'autre de la Corse pour former ensuite le Courant Nord Méditerranéen (CNM) également appelé courant liguro-provençal. Le CNM est composé principalement [8] de MAW, mais il entraîne aussi les eaux formées localement en hiver (WIW, Winter Intermediate Water [16]) et parfois de l'eau sous-jacente, en quantité variable mais non négligeable (figure $1 b$ ), notamment LIW (Levantine Intermediate Water) originaire du bassin levantin et, plus rarement, MDW (Mediterranean Deep Water). Le CNM est marqué par une activité à mésoéchelle qui croît durant l'automne et l'hiver, et persiste en été $[7,25]$. Cette activité se traduit par le développement de méandres et de tourbillons et par le passage d'ondes internes dans le sens transverse ou dans le sens parallèle à l'écoulement général [39].

Outre l'aspect descriptif visant à mieux comprendre la variabilité saisonnière et à méso-échelle de l'hydrodynamisme de la zone, cette étude est focalisée sur l'estimation des flux annuels des principales formes de l'azote (minérale, particulaire et organique dissoute) transitant à l'entrée du golfe du Lion, avant toute influence rhoda- a)

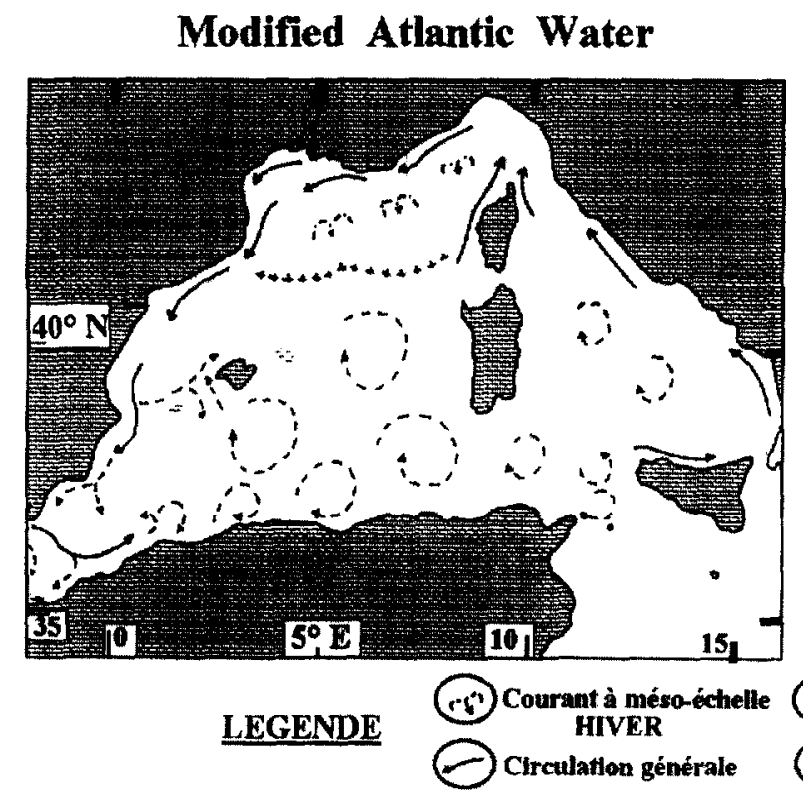

b)

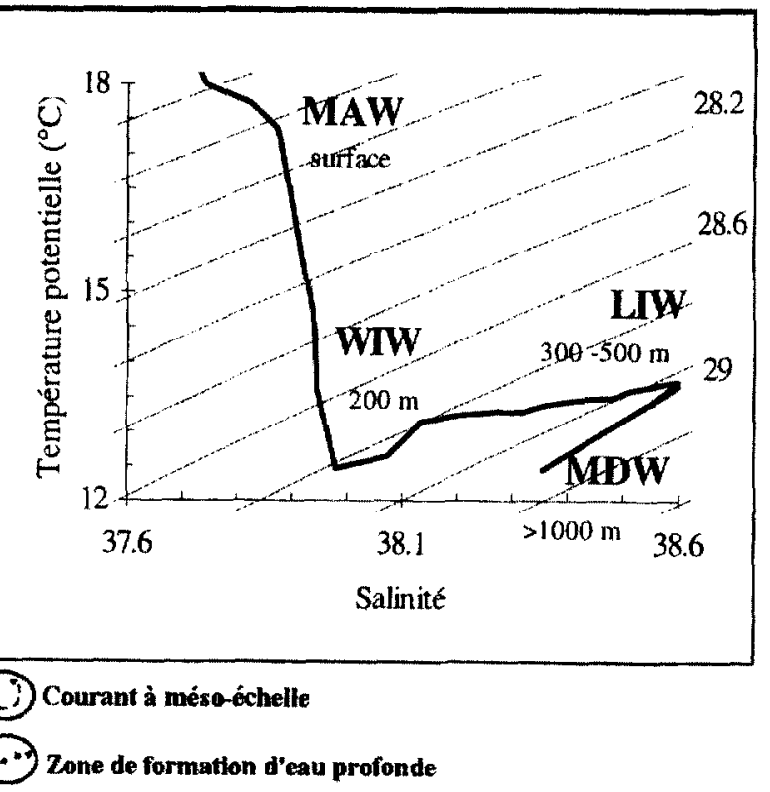

Figure 1. a) Circulation des eaux de surface en Méditerranée occidentale et principales zones de convection thermo-haline (d'après Millot [25]). b) Distribution type des masses d'eau rencontrées en été dans le golfe du Lion sur un diagramme $\theta / S$ (MAW = Modified Atlantic Water, WIW = Winter Intermediate Water, LIW = Levantine Intermediate Water, MDW = Mediterranean Deep Water, pointillés gris = isopycnes). La profondeur approximative du cœur de la masse d'eau est précisée.

Figure 1. a) Sea water surface circulation in the western part of the Mediterranean Sea and main zone of thermo-haline convection (after Millot [25]). b) Distribution of the water masses encountered in the Gulf of Lions, on a $0 / \mathrm{S}$ diagram (MAW = Modified Atlantic Water; WIW = Winter Intermediate Water, LIW = Levantine Intermediate Water, MDW = Mediterranean Deep Water, grey dashed lines $=$ isopycnes $)$. The approximate depth of the water mass core is also indicated. 
nienne. Les parts potentielles relatives des apports hauturiers et rhodaniens en termes de fertilisation de la zone du golfe du Lion sont ensuite évaluées en relation avec la production primaire présentée dans Conan et al. [9].

\section{MATÉRIELS ET MÉTHODES}

Un suivi mensuel de variables physiques et chimiques a été effectué entre octobre 1992 et octobre 1994 (figure 3 pour les dates), le long d'une radiale $\mathrm{N}-\mathrm{S}\left(5^{\circ} 12^{\prime} 5 \mathrm{E}\right.$ entre $43^{\circ} 10^{\prime} \mathrm{N}$ et $42^{\circ} 50^{\prime} \mathrm{N}$ ) au large de Marseille (figure 2). À chaque station, des profils hydrologiques verticaux (température, salinité, masse volumique) ont été réalisés à l'aide d'une bathysonde de type Seabird $911+$. Les caractéristiques données par le constructeur sont : pression (précision de $\pm 0,30 \mathrm{~m}$ à $10000 \mathrm{~m}$, résolution de $\pm 0,045 \mathrm{~m}$ ), température (précision de $\pm 0,0004{ }^{\circ} \mathrm{C}$, résolution de $\pm 0,0003{ }^{\circ} \mathrm{C}$ ), conductivité (précision de $\pm 0,00007 \mathrm{~S} \mathrm{~m}^{-1}$, résolution de $\pm 0,00004$ $\mathrm{S} \mathrm{m}^{-1}$ ). La composante barocline du courant est calculée à partir des anomalies de hauteur dynamique entre les stations selon les hypothèses géostrophiques. La vorticité planétaire est supposée constante compte tenu de la faible variation de latitude au long de la radiale. La résolution horizontale du courant est définie par la distance entre les
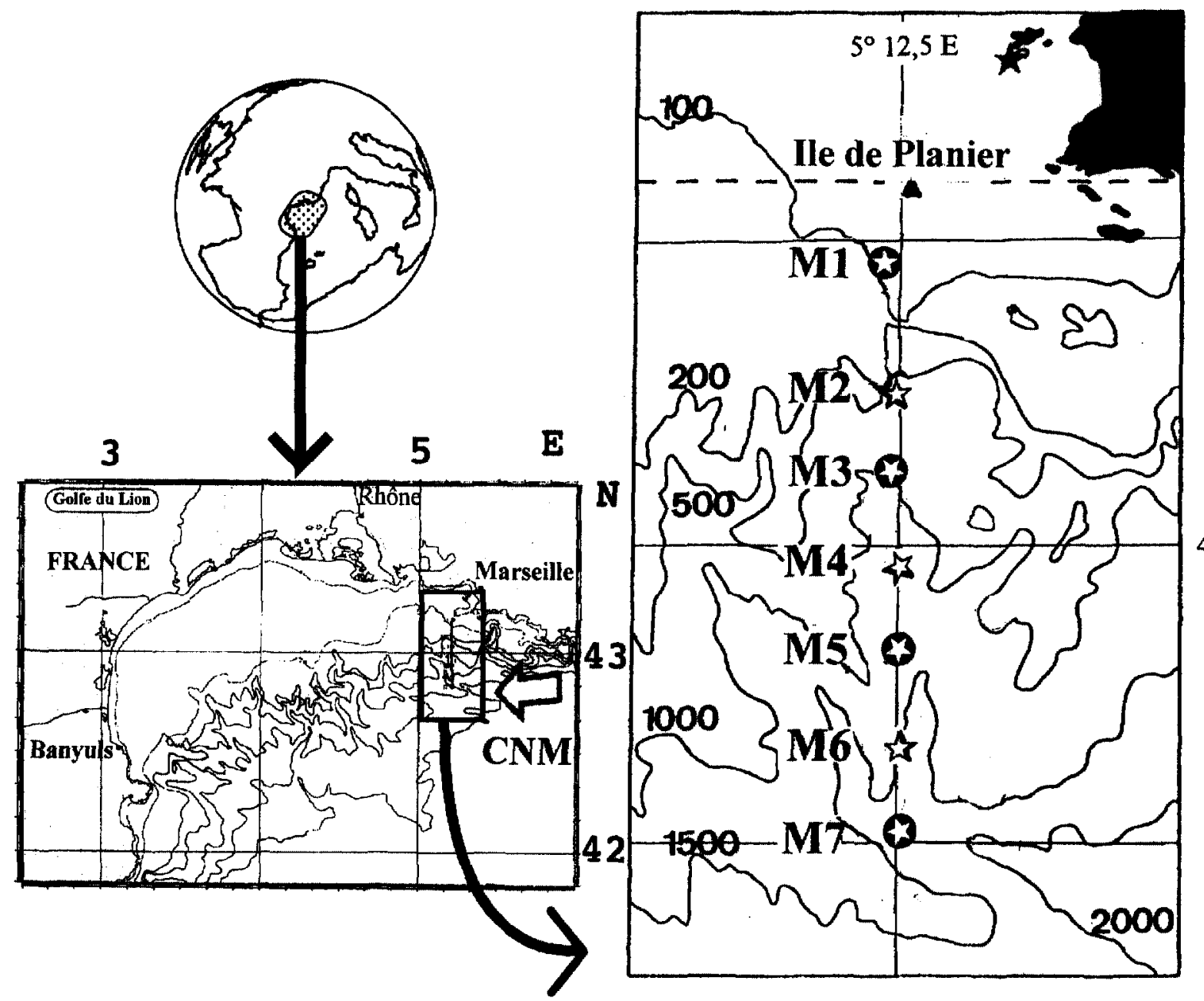

Figure 2. Localisation géographique de la zone d'échantillonnage, de la radiale prospectée et des stations échantillonnées dans le cadre du PNOC. CNM : Courant Nord Méditerranéen (circulation générale).

Figure 2. Geographical location of the sampled area, of the prospected transect and sampled stations in the framework of the PNOC. CNM $=$ Courant Nord Méditerranéen (North Western Mediterranean Current, i.e. general circulation). 


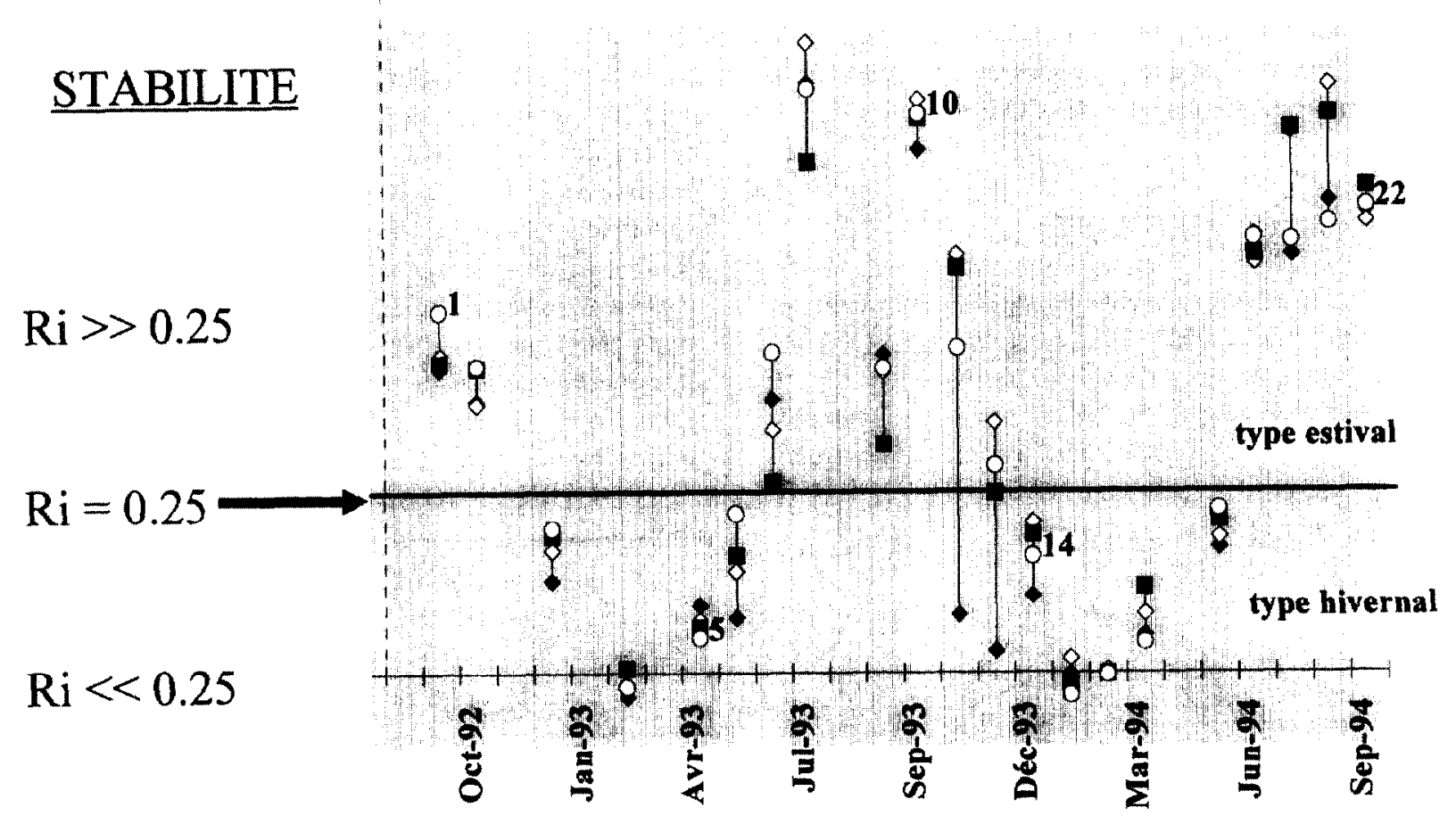

Figure 3. Stabilité de la colonne d'eau au cours des différentes sorties (numérotées de 1 à 22), obtenue à partir du nombre de Richardson (Ri) calculé aux stations impaires de la radiale $(\diamond) \mathrm{M} 1,(\diamond) \mathrm{M} 3,(\mathbb{\square}) \mathrm{M} 5$, (O) M7. Pour Ri supérieur à 0,25 , la colonne d'eau est considérée comme stable et de type hydrologique estival ; pour Ri inférieur à 0,25 elle est instable et qualifiée de type hydrologique hivernal.

Figure 3. Stability of the water column for each cruise (I to 22) estimated by the calculation of the Richardson number (Ri) at odd-numbercd

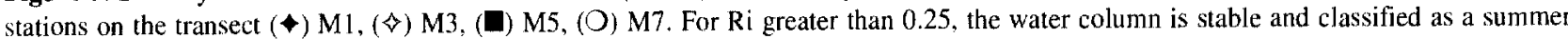
hydrological structure. For Ri lower than 0.25 it is considered as unstable and classified as a winter hydrological structure.

stations (environ 3 miles) et la résolution verticale, par l'épaisseur choisie pour les couches successives $(10 \mathrm{~m}$ entre 0 et $400 \mathrm{~m}, 20 \mathrm{~m}$ ensuite jusqu'à $600 \mathrm{~m}$ et $50 \mathrm{~m}$ audelà). L'erreur sur les vitesses du courant est fonction de la composante barotrope de ce dernier. Les équations sont données en fonction d'une vitesse de référence $\left(\mathrm{v}_{\mathrm{r}}\right)$, supposée nulle à $1000 \mathrm{~m}$ de profondeur [31]. La présence du littoral tend à éloigner le courant réel des conditions géostrophiques. La méthode d'approximation adoptée est décrite par Fieux [15] et consiste à extrapoler les surfaces isopycnes dans une couche d'eau fictive sous les stations continentales, avec conservation de la pente des isopycnes. Les incertitudes sur les estimations des vitesses $\left(\Delta \mathrm{U}= \pm 1 \mathrm{~cm} \mathrm{~s}^{-1}\right)$ et des flux $(\Delta \mathrm{F}= \pm 0,1 \mathrm{~Sv})$ sont tirées des travaux de Byun [5] en Atlantique du N-E et adaptées à la Méditerranée par Durrieu de Madron [13]. Une vitesse de $5 \mathrm{~cm} \mathrm{~s}^{-1}$ est choisie comme limite inférieure pour les vitesses du CNM.
Des prélèvements d'eau de mer sont effectués à douze profondeurs entre 0 et $200 \mathrm{~m}$, aux stations impaires de la radiale (figure 2) à l'aide d'une rosette Seabird de douze bouteilles Niskin $(8 \mathrm{~L})$. Des sous-échantillons de $10 \mathrm{~mL}$ d'eau de mer sont prélevés dans des flacons à scintillation en polyéthylène et ont été immédiatement congelés. À l'arrivée au laboratoire, ces échantillons non filtrés sont rapidement décongelés et analysés pour la mesure des concentrations en nitrate $\left(\mathrm{NO}_{3}\right.$, erreur standard $\pm 0,10 \mu \mathrm{M})$ et en nitrite $\left(\mathrm{NO}_{2}\right.$, erreur standard $\left.\pm 0,02 \mu \mathrm{M}\right)$ selon la méthode de Wood et al. [45], automatisées sur un analyseur de type Technicon selon le protocole défini par Tréguer et Le Corre [43].

Des sous-échantillons de $250 \mathrm{~mL}$ d'eau de mer sont également prélevés dans des flacons en polyéthylène opaques et immédiatement filtrés sur filtre Whatman GF/F de $25 \mathrm{~mm}$ de diamètre. Les filtres sont ensuite rapidement congelés. Au laboratoire, ces filtres permettent de 
déterminer les concentrations en chlorophylles totales à l'aide d'un fluorimètre Turner Designs de type 10.005R, selon la procédure fluorimétrique définie par Holm-Hansen et Riemann [21].

L'azote total $(\mathrm{Nt})$ et l'azote particulaire (NP) sont déterminés selon la méthode préconisée par Pujo-Pay et Raimbault [38], modifiée pour permettre l'analyse simultanée de l'azote organique dissous (NOD). La méthode consiste en une minéralisation par oxydation humide en présence de persulfate $\left(30 \mathrm{~min}\right.$ à $\left.120^{\circ} \mathrm{C}\right)$, de $40 \mathrm{~mL}$ d'eau de mer pour la mesure de $\mathrm{Nt}$ et d'un filtre précalciné $(4 \mathrm{~h}$ à $450{ }^{\circ} \mathrm{C}$ ) Whatman $\mathrm{GF} / \mathrm{F}$ de $25 \mathrm{~mm}$ de diamètre (sur lequel ont été filtrés $250 \mathrm{~mL}$ d'échantillon sous faible dépression $<50 \mathrm{~mm} \mathrm{Hg}$ ) dans $20 \mathrm{~mL}$ d'eau déionisée pour NP. Les formes minérales majoritaires (justifiées dans [37]) et NP sont ensuite soustraits à Nt pour fournir une estimation de la concentration en $\mathrm{NOD}: \mathrm{NOD}=\mathrm{Nt}-$ $\mathrm{PN}-\mathrm{NO}_{3}-\mathrm{NO}_{2}$.

Les transports en élément à travers la radiale sont obtenus par la somme globale des flux intermédiaires, déduits du produit du flux en eau et de la concentration de l'élément considéré entre chaque couple de stations, par couches successives sur la verticale [7]. Les résultats correspondent au passage de matière à travers l'ensemble de la radiale, indépendamment de la présence ou de l'absence du CNM défini précédemment pour des vitesses supérieures à $5 \mathrm{~cm} \mathrm{~s}^{-1}$.

\section{RÉSULTATS}

Le classement des campagnes à la mer en deux saisons hydrologiques, hiver et été, est fondé sur le coefficient de Richardson (Ri) mesurant la stabilité relative d'une colonne d'eau (figure 3). La rupture des gradients thermo-halins s'effectue par des mouvements turbulents sous l'effet de vagues formées à l'interface de ces gradients par les vents, les vagues de surface, les fluctuations de la pression atmosphérique ou l'écoulement des eaux sur les irrégularités du fond. Cette instabilité dite de Kelvin-Helmholtz a été utilisée en Méditerranée pour décrire la rupture de la thermocline en automne [46]. Lorsque $\mathrm{Ri}$ est élevé $(>0,25)$, la structure physique est plutôt stable, de type «été hydrologique ». À l'inverse, lorsque Ri est faible $(<0,25)$, la structure physique est instable, de type «hiver hydrologique ». Ce critère de stabilité permet ici de regrouper les 22 sorties en cinq groupes successifs (figure 3). Trois groupes s'inscrivent en période d'été hydrologique, entre mai et novembre (sorties 1 et 2,7 à
12 et 19 à 22), alors que deux groupes caractérisent la période d'hiver hydrologique de novembre à mai (sorties 3 à 6 et 13 à 18). L'hiver hydrologique et l'été hydrologique intègrent les quatre saisons classiquement reconnues (hiver, printemps, été, automne).

\subsection{Variabilité du système hydrodynamique}

Les vitesses maximales rencontrées au cours de chaque sortie rendent compte de la variabilité saisonnière classique du CNM et confirment la dualité de la structure dynamique décrite par Millot [25]. Pendant le suivi, les vitesses maximales calculées à travers la radiale varient entre 45 et $60 \mathrm{~cm} \mathrm{~s}^{-1}$ en période hivernale et entre 15 et $30 \mathrm{~cm} \mathrm{~s}^{-1}$ en période estivale (figure $4 a$ ). Ces vitesses maximales sont généralement associées à une structure typique du courant. En période hivernale, le CNM est caractérisé par une veine bien définie, plutôt étroite $(-30 \mathrm{~km})$ et profonde $(350-400 \mathrm{~m})$ (figure $4 b)$ dont le cour est localisé près de la côte, au-dessus de la pente continentale ( $\sim 20 \mathrm{~km}$ de l'île du Planier). Le courant présente alors une allure typique en forme de jet [35]. Les vitesses hivernales du courant sont marquées par deux maximums (figure 4a), l'un en début (novembre à décembre) et l'autre en fin de période d'hiver hydrologique (avril à mai), voire en début du mois de juin (en 1994). Au cour de l'hiver (janvier à mars), les vitesses maximales du courant sont sensiblement plus faibles qu'au cours de ces deux événements. Pendant l'été hydrologique, l'allure générale du courant évolue vers une forme plus diffuse, la veine tend à s'élargir $(>40 \mathrm{~km})$ et son extension en profondeur diminue $(-200 \mathrm{~m})$; le coeur de ce courant $\mathrm{sc}$ trouvc alors très au large, voire au-delà de la station $\mathrm{M} 7$ (figure $4 \mathrm{c}$ ).

Les structures typiques du courant que nous venons de décrire sont fréquemment perturbées par de fortes variations de la vitesse (entre campagnes à la mer successives, figure $4 a$ ), de la forme et de la position de la veine et du cceur du courant. Par exemple, il est possible de rencontrer un coeur et une veine de courant à plus de $40 \mathrm{~km}$ au large en hiver (figure $4 d$ ), ou une veine de courant bien définie et très proche de la côte en été (figure $4 e$ ). Les oscillations horizontales de la veine peuvent dépasser $30 \mathrm{~km}$ en $15 \mathrm{j}$, et l'extension verticale du CNM peut varier rapidement comme l'illustrent les campagnes du 19 juin 1993 et du 2 juillet 1993 (figures $4 e, 4 c$ ). D'importantes modifications du courant sont également intervenues entre la première et la seconde partie du suivi (H92 par rapport à H93, et E93 par rapport à E94), cette 
A)

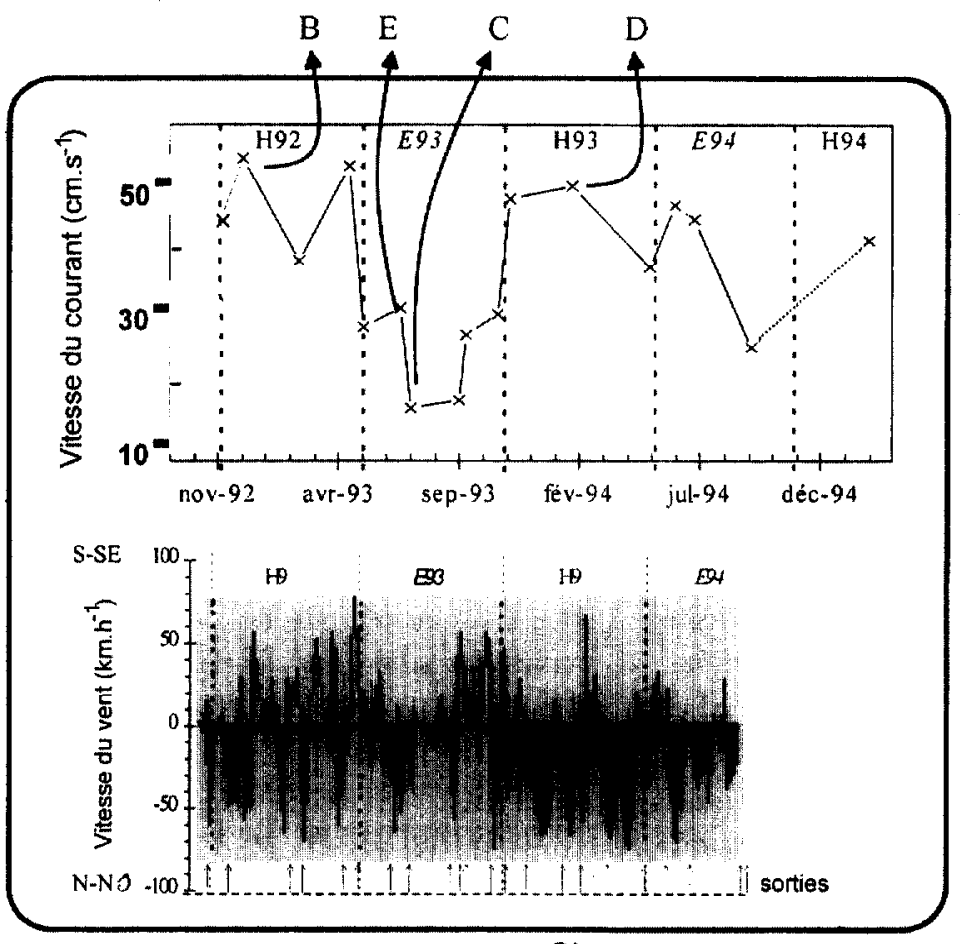

B)

HIVER

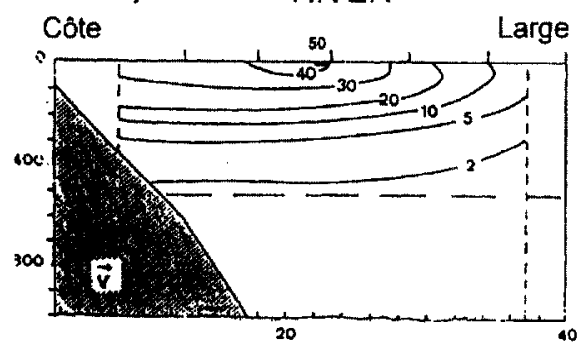

DISTANCE DE PLANIER. $\mathrm{km}$

D) 22 janvier 1994

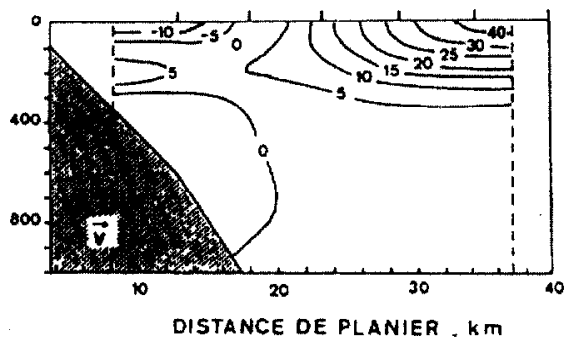

C)

ETE

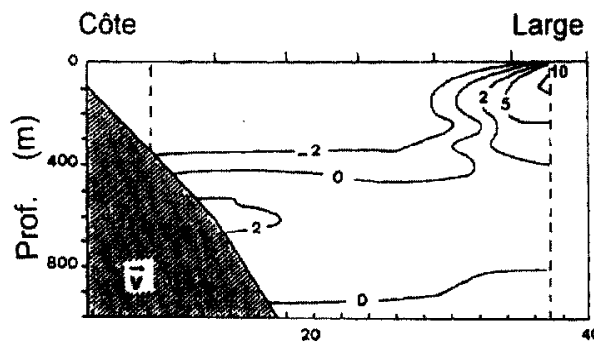

distance de planier * $\mathrm{km}$

E) 19 juin 1993

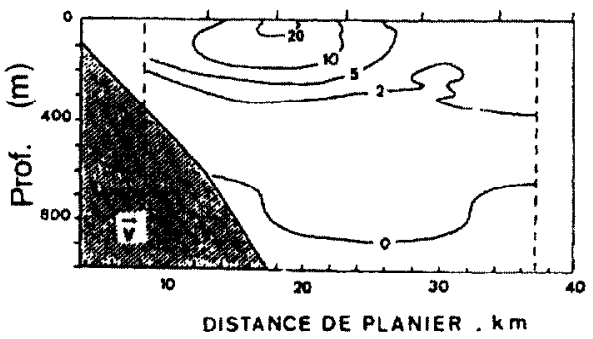

Figure 4. A) Évolution temporelle de la vitesse maximale du courant sur la radiale M1-M7. Les flèches et les lettres repèrent la position des campagnes utilisées pour la représentation des isotachs de courant ci-après. En dessous, évolution temporelle de la forec et de la direction du vent classé selon deux directions principales S-SE et N-NO (vitesses et directions moyennées sur cinq jours ; données Météo-France).

Distribution côte-large des isotachs le long de la radiale $\mathrm{M} 1-\mathrm{M} 7$. B) Allure typique de la veine du CNM en situation hydrologique hivernale classique (décembre 1992). C) Allure typique de la veine du $\mathrm{CNM}$ en situation hydrologique estivale classique (juin 1993). D) Variabilité rencontrée au cours du suivi : la structure dynamique du 22 janvier 1994 s'éloigne de la structure classique hivernalc. E) Variabilité rencontrée au cours du suivi : la structure dynamique du 19 juin 1993 s'éloigne de la structure classique estivale.

Figure 4. A) Temporal evolution of the maximum curtent speed on the MI-M7 transect. The arrows and the letters indicate the position of the cruises used in the representation of the current isotachs above. Below this graph, temporal evolution of the wind speed and direction according to two main directions (S-SE and N-NW, average on five days; data from Meteo-France).

On-offshore representation of the isotachs of the CNM along the M1-M7 transect. B) Typical shape of the current in a hydrological winter situation (December 1992). C) Typical shape of the current in a hydrological summer situation (June 1993). D) Variability during the time series: the dynamic structure of the 22nd January 1994 is far from the typical winter structure. E) Variability during the time series: the dynamic structure of the 19 th June 1993 is far from the typical summer structure 
dernière étant caractérisée par un courant plus fort et plus près des côtes. Une modification importante au cours des deux années du suivi a également été observée dans le régime des vents (figure $4 a$ ). Durant la première partie du suivi (H92 et E93), les vents ont soufflé alternativement et de façon équilibrée entre les secteurs $\mathrm{S}-\mathrm{SE}$ et $\mathrm{N}-\mathrm{NO}$ ( $\sim 50 \%$ dans chaque cadran). Au cours de la seconde année du suivi (H93 et E94), les vents de secteur N-NO ont été largement dominants $(>60 \%)$ et plus violents que lors de la première année.

Les formations d'eau profonde par convections thermohalines se localisent dans des zones plus au large que la station M7 (figures 1 et 2 ) et n'ont donc pas d'influence directe sur les coupes réalisées au cours du suivi. En revanche, nous avons pu identifier trois événements dynamiques importants pour la zone d'étude et pour le fonctionnement de l'écosystème [7]. Il s'agit des formations d'eau d'hiver (WIW) sur le plateau continental et au large (non détaillées dans la présente étude), des apports d'eau dessalée d'origine continentale et des remontées d'eau profonde le long de la pente continentale. La campagne en mer effectuée le 30 octobre 1992 offre une image synthétique de l'effet des deux phénomènes cités précédemment (figure 5).

Des eaux d'origine continentale sont identifiées entre les stations M2 et M4 par une bulle de salinité inférieure à 37,10 (masse volumique $<27,00$ ). L'isohaline 37,80 pourrait servir de limite arbitraire à ces apports probablement d'origine rhodanienne compte tenu des quantités d'eau impliquées et malgré la position de la radiale à l'est de l'embouchure du Rhône. La limite la plus au large de cette bulle correspond à la limite interne du CNM qui se situe très au large au cours de cette sortie (figure 5). Ces apports d'eau dessalée ont une origine relativement récente comme le confirme la faible salinité mesurée et la présence d'une concentration non négligeable en nitrate $(0,5$ à $0,7 \mu \mathrm{M})$. La consommation biologique de ces sels nutritifs ne semble pas achevée malgré les fortes concen-
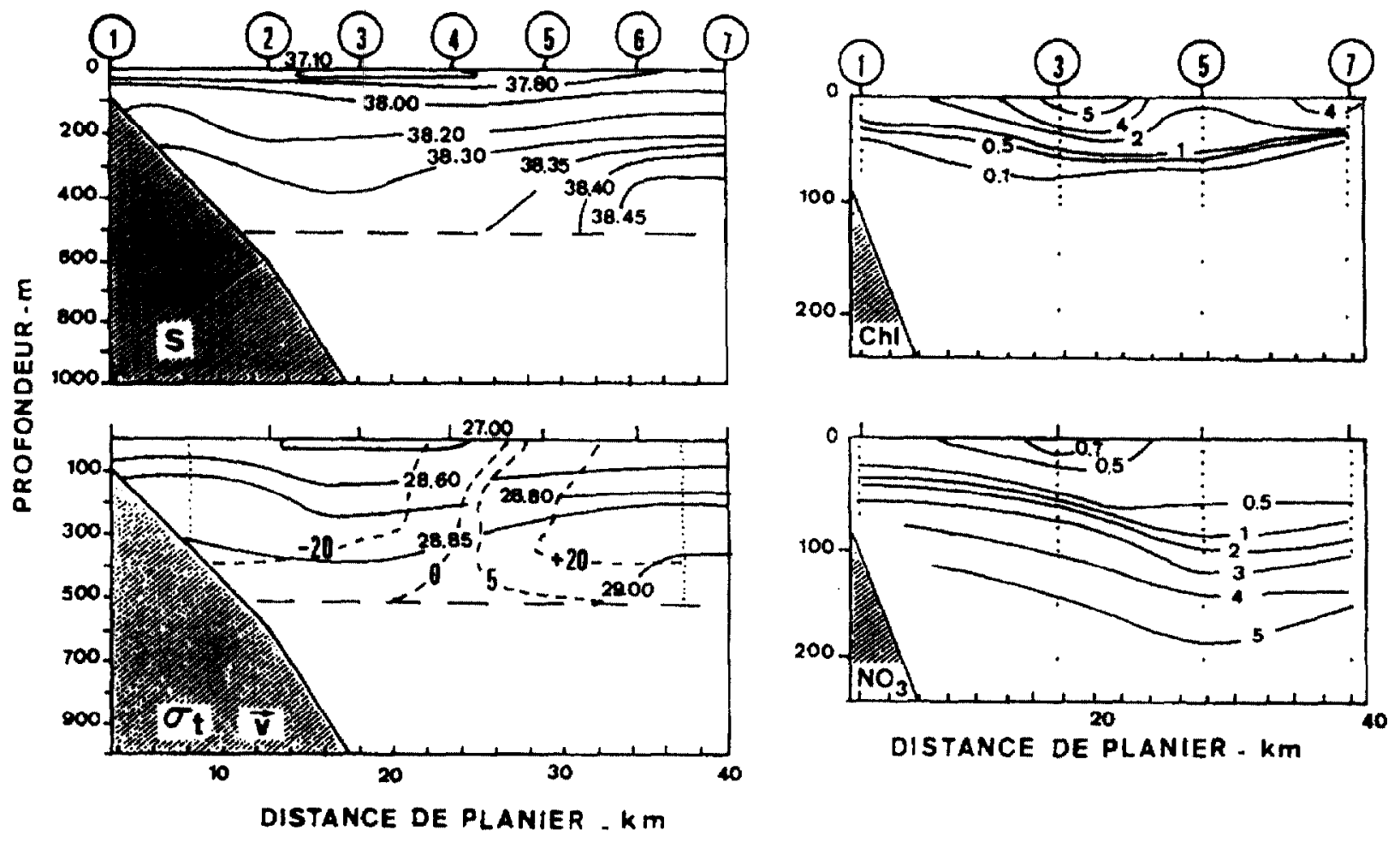

Figure 5. Coupes verticales le long de la radiale M1-M7, 30 octobre 1992, de salinité (S), sigma (ligne continue ot) et vitesse du courant géostrophique (en pointillés : v en $\mathrm{cm} \mathrm{s}^{-1}$ ). $\AA$ droite, chlorophylle totale (chl en $\mathrm{mg} \mathrm{m}^{-3}$ ) et nitrate $\left(\mathrm{NO}_{3}\right.$ en $\mu \mathrm{M}$ ).

Figure 5. Vertical transects along the M1-M7 transect, 30th October 1992: salinity (S), sigma (full line ot) and geostrophic velocity (dashed line: $v$ in $\mathrm{cm} \mathrm{s}^{-1}$ ). On the right, total chlorophyll ( $\mathrm{chl}$ in $\mathrm{mg} \mathrm{m}^{-3}$ ) and nitrate $\left(\mathrm{NO}_{3}\right.$ in $\left.\mu \mathrm{M}\right)$. 
trations en chlorophylles mesurées dans le cœur de cette bulle (>5,0 $\mathrm{mg} \mathrm{m}^{-3}$ ) et dans la zone frontale définie par cette dernière $\left(4,0 \mathrm{mg} \mathrm{m}^{-3}\right.$ à la station $\left.\mathrm{M} 7\right)$.

Plus en profondeur (entre 60 et $400 \mathrm{~m}$ ) le long de talus continental (entre les stations M1 et M3), les isohalines comprises entre 38,00 et 38,30 et les isopycnes comprises entre 28,60 et 28,85 s'incurvent vers la surface et remontent le long de la pente continentale. L'allure de ces isolignes contraste avec la structure en forme de dôme généralement observée sur la radiale [7]. Elle caractérise une remontée d'eau le long de la pente continentale; dans ce cas, le cœur de LIW limité par l'isohaline 38,45 est localisé au large (station M7) et s'éloigne de la pente continentale [8]. Les eaux de la remontée proviennent d'une profondeur d'environ $500 \mathrm{~m}$. Elles sont pauvres en chlorophylle mais plutôt riches en sels nutritifs comme le confirment les isoplètes 0,5 à $5,0 \mu \mathrm{M}$ de nitrate atteignant le plateau continental (figure 5).

\subsection{Flux à travers la radiale}

En raison de la stratégie d'échantillonnage adoptée et de la forte variabilité spatiale rencontrée, le CNM n'a pas pu être traversé totalement lors de chaque sortie. Le bord externe du courant était fréquemment situé plus au large. 11 est important de noter que la valeur des flux proposée dans cette étude n'est qu'une estimation des flux d'azote transitant à l'entrée du golfe du Lion entre $43^{\circ} 10^{\prime} \mathrm{N}$ et $42^{\circ} 50^{\prime} \mathrm{N}$ entre les stations $\mathrm{M} 1$ et $\mathrm{M} 7$ de la radiale prospectée et ne peut constituer qu'une sous-estimation des flux réels du CNM.

Le flux en eau à travers la radiale peut varier entre 0 et plus de $1,5 \pm 0,1 \mathrm{~Sv}$ au cours de l'année $(1,8 \pm 0,1 \mathrm{~Sv}$ durant l'hiver 1991), soit en moyenne des valeurs de 0 à $0,05 \pm 0,01 \mathrm{~Sv} \mathrm{~km}{ }^{-1}$ (figure $6 a$ ). Le flux en eau est maximal pendant la période hydrologique hivernale (supérieur à $1,0 \pm 0,1 \mathrm{~Sv}$ ) et marqué par deux maximums, correspondant aux vitesses maximales du courant, décrits précédemment. Le flux en eau diminue ensuite rapidement au cours de la période hydrologique estivalc, pouvant même atteindre des valeurs négligeables pendant le mois de juillet (figure $6 a$ ). La distribution verticale du débit par couche indique que les deux maximums hivernaux affectent principalement la couche de surface 0-100 m. Dans la couche profonde $(100-200 \mathrm{~m})$, les maximums sont rencontrés en décembre ou janvier après une augmentation régulière et sont suivis par une décroissance plus ou moins régulière (figure $6 a$ ).
En terme de flux de nitrate, l'évolution de la quantité transportée à travers la radiale est en relation directe avec le cycle saisonnier des éléments nutritifs sur la colonne d'eau [9]. En période hydrologique hivernale, le flux en nitrate atteint 6,0 à $8,0 \pm 0,8 \mu \mathrm{g} \mathrm{m}^{-3} \mathrm{~s}^{-1}$, mais il est inférieur à $2,0 \pm 0,2 \mu \mathrm{g} \mathrm{m}^{-3} \mathrm{~s}^{-1}$ en période hydrologique estivale (figure $6 b$ ). Conformément à la distribution verticale classique des sels nutritifs, ce flux est maximal dans la couche profonde $(100-200 \mathrm{~m})$ et reste faible dans la couche de surface tout au long de l'année. Le flux en azote particulaire (NP) est le plus élevé en période d'hiver hydrologique (figure $6 b$ ) avec des valeurs supérieures à $1,0 \pm 0,1 \mu \mathrm{g} \mathrm{m}^{-3} \mathrm{~s}^{-1}$; il est faible durant l'été hydrologique $\left(<0,2 \pm 0,1 \mu \mathrm{g} \mathrm{m}^{-3} \mathrm{~s}^{-1}\right)$. Le flux en NP est observé essentiellement dans la couche de surface $(0-100 \mathrm{~m})$ et reste toujours faible dans la couche plus profonde, conformément à la distribution des concentrations en NP qui diminuent fortement au-delà de $100 \mathrm{~m} \mathrm{[36].}$

La quantité totale d'azote transitant annuellement à travers la radiale, entre 0 et $200 \mathrm{~m}$ de profondeur, s'élève à $72 \pm 8 \mathrm{kTa}^{-1}(39 \%)$ sous forme de nitrate $\left(\mathrm{NO}_{3}\right)$, dont près de $30 \%$ dans la couche superficielle $0-100 \mathrm{~m}$, et entre 10 et $14 \mathrm{kT} \mathrm{a}^{-1}(6 \%)$ sous forme NP, mais $80 \%$ de cette quantité transite dans la couche superficielle 0 $100 \mathrm{~m}$ ( tableau I). La majorité de l'azote est donc transportée sous forme d'azote organique dissous (NOD), soit $103 \pm 11 \mathrm{kT} \mathrm{a}^{-1}(55 \%)$. Les autres formes de l'azote (nitrite, ammonium, urée...) sont intégrées dans ce dernier compartiment mais leurs concentrations sont d'un

Tableau I. Bilan annuel des flux de matière calculés à travers la radiale $\mathrm{M} 1-\mathrm{M} 7$, dans la couche $0-100 \mathrm{~m}$ et $0-200 \mathrm{~m}$ en nitrate $\left(\mathrm{NO}_{3}\right)$, azote particulaire (NP), azote organique dissous (NOD) et azote total $(\Sigma)$ exprimé en $\mathrm{kT} \mathrm{a}^{-1}$. Les flux d'azote apportés par le Rhône sont également indiqués, ainsi que les pourcentages moyens (\%) de chaque forme dans le flux total.

Table I. Annual budget of calculated matter transports across the $\mathrm{M} 1-\mathrm{M} 7$ transect into the $0-100 \mathrm{~m}$ layer and the $0-200 \mathrm{~m}$ layer of nitrate $\left(\mathrm{NO}_{3}\right)$, particulate nitrogen (NP), dissolved organic nitrogen (NOD) and total nitrogen $(\Sigma)$ expressed in $\mathrm{kT} \mathrm{a}^{-1}$. Nitrogen fluxes injected by the river Rhone are also indicated, as the mean contribution of each form. $(\%)$ in the total flux.

\begin{tabular}{lrrrrrr}
\hline & \multicolumn{2}{c}{ Radiale MI -M7 } & \multicolumn{2}{c}{ Rhône } \\
\hline kT a $^{\mathbf{1}}$ & $\mathbf{0 - 2 0 0} \mathbf{~ m}$ & \%moyen & $\mathbf{0 - 1 0 0} \mathbf{~ m}$ & \%moyen & & \%moyen \\
\hline $\mathrm{N}_{3}$ & $72 \pm 8$ & 39 & $25 \pm 3$ & 26 & $64 \pm 7$ & 80 \\
$\mathrm{NP}$ & $12 \pm 2$ & 6 & $10 \pm \mathrm{I}$ & 10 & $7 \pm 1$ & 9 \\
$\mathrm{NOD}$ & $103 \pm 11$ & 55 & $62 \pm 7$ & 64 & $9 \pm 1$ & 11 \\
$\Sigma$ & $187 \pm 20$ & 100 & $97 \pm 10$ & 100 & $80 \pm 8$ & 100 \\
\hline
\end{tabular}


A)

\section{Couche:}

$\square$ 0-200m

$100-200 \mathrm{~m}$

0-100m

\section{Echelles ci-dessous:}

g. $\mathrm{m}^{-3} \cdot \mathrm{s}^{-1} \quad$ (courbe)

T.s (histogramme)

B)

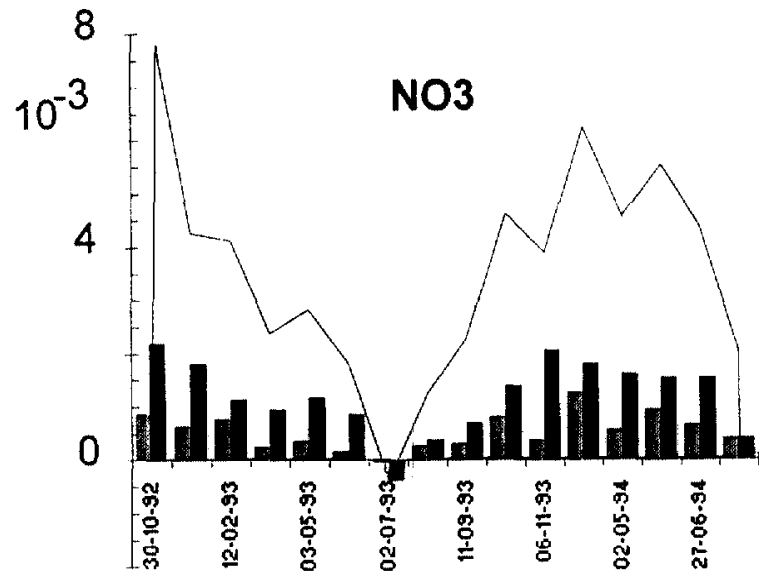

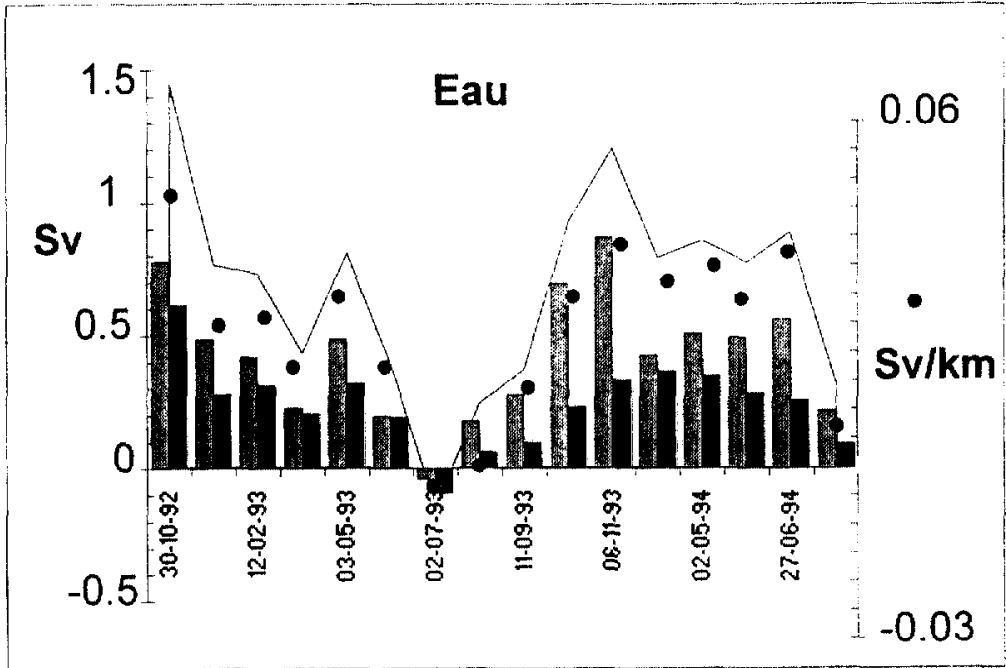

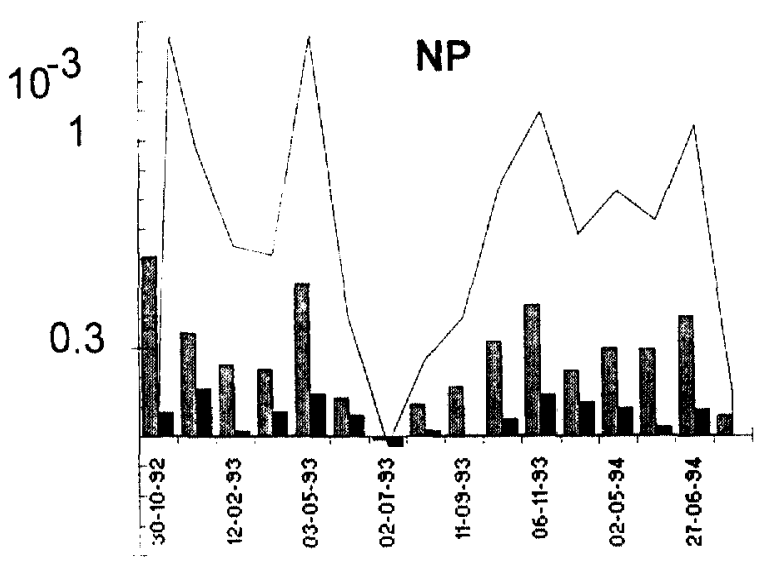

Figure 6. Les aires représentent l'évolution à travers la radiale M1-M7 sur une couche (0-200 m : A) tlux en eau (Sv) (les points (O) correspondent au flux en $\mathrm{Sv} \mathrm{km^{-1 }}$ sur l'échelle de droite). B) flux en nitrate $\left(\mathrm{NO}_{3} ; \mathrm{g} \mathrm{m}^{-3} \mathrm{~s}^{-1}\right)$, et en azote particulaire $\left(\mathrm{NP} ; \mathrm{g} \mathrm{m}^{-3} \mathrm{~s}^{-1}\right)$. Les histogrammes expriment les flux selon deux couches successives $\left(0-100 \mathrm{~m}\right.$ et $100-200 \mathrm{~m}$ ), en Sv pour $\mathrm{A}$ ) et en $\mathrm{T} \mathrm{s} \mathrm{s}^{-1}$ pour B).

Figure 6. The areas represent the evolution across the M1-M7 transect in the (0-200 $\mathrm{m}$ layer of: A) Water fluxes (Sv) ((O) refers to the flux (Sv km$\left.{ }^{-1}\right)$ on the right scale) and B) Nitrate flux $\left(\mathrm{NO}_{3}: \mathrm{g} \mathrm{m}^{-1} \mathrm{~s}^{-1}\right)$ and particulate nitrogen flux $\left(\mathrm{NP} ; \mathrm{g} \mathrm{m}^{-1} \mathrm{~s}^{-1}\right)$. The histograms are representative of the same fluxes for successive layers $(0-100 \mathrm{~m}$ and $100-200 \mathrm{~m})$ in $S v$ for $A$ ) and $T s^{-1}$ for $B$ ).

ordre de grandeur de dix à cent fois inférieur à la concentration du NOD, ce qui minimise l'erreur commise sur les estimations du flux. La quantité totale d'azote apportée par le flux advectif de la circulation générale dans le golfe du Lion, à travers la radiale M1-M7, dans la couche d'eau superficielle 0-200 m, est de $187 \pm 20 \mathrm{kT} \mathrm{a}^{-1}$. Ce flux se répartit équitablement sur la verticale puisqu'en moyenne $50 \%$ de ce flux se situe dans la couche 0$100 \mathrm{~m}$, soit $97 \pm 10 \mathrm{kT} \mathrm{a}^{-1}$ (tableau I).

\section{DISCUSSION}

La Méditerranée nord-occidentale présente d'importantes analogies hydrodynamiques et fonctionnelles avec les parties nordiques d'autres mers plus ou moins fermées. comme la Méditerranée orientale ou la mer asiatique [26]. Il est donc important de bien comprendre et de modéliser le rôle de la circulation générale dans les problèmes de fertilisation des zones continentales [24] afin 
de réduire les incertitudes concernant les bilans de productivité à l'échelle des bassins.

\subsection{Source d'erreur des estimations}

L'analyse et les calculs effectués dans cette étude reposent sur la validité des hypothèses géostrophiques appliquées au CNM. Bien que ce courant réponde de manière satisfaisante à ces critères [31], la stratégie d'échantillonnage adoptée ici, i.e. prospection régulière d'un transect en zone côtière, peut être une source d'erreur importante. De plus, l'échantillonnage, discret dans le temps et dans l'espace, est réalisé lorsque les conditions météorologiques sont favorables. Il est possible que les maxima observés correspondent à une estimation plus ou moins importante et variable des caractéristiques réelles de la circulation générale (vitesses et flux.).

Compte tenu de ces restrictions, mais également du fait que le CNM n'a pu être échantillonné en totalité lors de chaque campagnc, l'cstimation des flux en azotc réalisćc doit être considérée comme représentative du transport de matière à travers la radiale $\mathrm{N}-\mathrm{S}$ échantillonnée, plutôt que comme le flux global en azote du CNM. L'intérêt des estimations de flux réside dans le fait que les quantités de matière qui transitent à travers la radiale sont susceptibles d'être introduites directement et rapidement sur le plateau continental. Cet azote est alors disponible pour les communautés biologiques côtières et participe à la productivité de la zone. Les ordres de grandeur de la vitesse et du flux en eau estimés sont proches et en accord avec les résultats de travaux portant sur le CNM, en mer Ligure $[1,35]$ ou en mer Catalane [17].

\subsection{Variabilité hydrologique saisonnière et interannuelle}

Crépon et Boukthir [11] et Astraldi et Gasparini [1] ont montré que la variabilité du CNM résulte de la combinaison de la variabilité des courants est et ouest Corse. Le premier maximum en période hydrologique hivernale (figures $4 a, 6 a$ ) est associé à une augmentation significative du flux du courant est Corse en relation avec le maximum d'écart thermique entre la mer Tyrrhénienne et la mer Ligure. Le second maximum, en fin de période hydrologique hivernale, correspond à une augmentation importante du flux du courant ouest Corse en réponse aux formations hivernales d'eau profonde (figure 1). En fonction de l'intensité de ces formations [20], le maximuru du
CNM en fin de période hydrologique hivernale est plus ou moins prononcé [7].

Les vents de secteur $\mathrm{N}-\mathrm{NO}$, plus fréquents, plus réguliers et plus intenses (hiver 1993, figure 4a) sont à l'origine d'une position plus hauturière du CNM [6]. L'action des vents de secteur S-SE est opposée [7] et tend à rapprocher les eaux superficielles de la côte [42]. Ces résultats confirment l'influence du vent sur la circulation générale superficielle [25] et sur le fonctionnement global de l'écosystème [24] dans le golfe du Lion.

La combinaison de l'effet du vent et des plongées d'eau en hiver fournit une explication satisfaisante à la variabilité intersaisonnière des vitesses calculées sur la radiale (figure 4a), maximales au cours du premier hiver (H92) par rapport au second (H93) et au cours du deuxième été (E94) par rapport au premier (E93). Au cours de H93, le cœur du CNM a été localisé en moyenne plus au large que lors de $\mathrm{H} 92$ [7]. Les vitesses maximales ont été sousestimées en raison de la position du cour au-delà de la station M7. Le rapprochement de la veine de courant de la côte (i.e. E94 par rapport à E93 ; figure $4 a$ ) a permis une plus grande probabilité de présence du cour sur la radiale et pourrait limiter le développement de méandres.

\subsection{Variabilité hydrologique à méso-échelle}

La veine de courant définie par le CNM est généralement caractérisée par de fortes variations de vitesse et de position. Ces variations répondent à des oscillations plus ou moins continues, pouvant être interprétées comme des mouvements ondulatoires créés par le passage de méandres ou d'ondes internes de grande amplitude $[8,25,39]$. À ces variations transverses à l'écoulement du courant, s'ajoutent des variations parallèles à l'écoulement du courant, engendrant de véritable pulses de la vitesse [39].

Ces variations sont à l'origine d'importants enrichissements en sels nutritifs [23], particulièrement dans les zones frontales $[24,35]$. Dans la zone de la radiale, il est possible d'identifier des apports en sels nutritifs sur le plateau continental par de l'eau d'origine continentale (Rhône) et par des remontées d'eau profonde d'origine hauturière (figure 5). Les apports d'eau dessalée interviennent en surface et impliquent des phénomènes de diffusion et de mélange pour rendre les sels nutritifs disponibles au système biologique marin [34]. Dans la zone d'étude les sels nutritifs sont généralement faiblement concentrés et le développement de la biomasse phytoplanctonique plutôt avancé (figure 5). Une colonne stratifiée permet un déplacement rapide des eaux de 
faible salinité ( «glissement » sur la thermocline en relation avec les vents). Les remontées d'eau profonde sont identifiées dans la zone d'étude par une augmentation significative des concentrations en sels nutritifs et par des concentrations plutôt faibles en chlorophylles (figure 5).

\subsection{Variabilité des flux en azote à travers la radiale}

Les transports en nitrate les plus importants sont mesurés en octobre ou novembre (figure $6 b$ ). Ces maximums ne coïncident pas nécessairement avec l'augmentation importante des flux en eau en début d'hiver hydrologique (comme par exemple lors de la seconde partie du suivi). En effet, l'augmentation et la variabilité du flux en nitrate sont plutôt à relier aux phénomènes de mélanges et d'enrichissement de la couche superficielle en sels nutritifs à travers ou lors de la rupture de la stratification. Le transport en nitrate dans la couche profonde $(100-200 \mathrm{~m})$ est relativement peu variable et la variabilité totale de la colonne d'eau est principalement en relation avec les variations dans la couche superficielle (figure $6 b$ ).

À la fin de la période hydrologique estivale, la couche superficielle 0-50 m est pratiquement épuisée en nitrate, mais les gradients subsuperficiels (sous la couche mélangée superficielle) se sont intensifiés tout au long de cette saison, par rapprochement de la surface d'eau plus dense et plus riche en sels nutritifs [7, 9]. En fonction de la position de ces gradients, l'accélération du CNM au début de l'hiver hydrologique, dans la couche $0-100 \mathrm{~m}$, peut entraîner cette eau riche en sels nutritifs (4,0 à $6,0 \mu \mathrm{M}$ en nitrate) et expliquer le maximum important de flux en nitrate (le 30/10/92), ou bien ne pas entraîner cette eau et n'intéresser alors que des eaux pauvres en nitrate $(6 / 11 / 93)$ (figure $6 b$ ). Au cours de l'hiver hydrologique, la couche mélangée peut atteindre des profondeurs de 200 à $500 \mathrm{~m}$ [7] mais les concentrations en nitrate dépassent rarcment 1,0 à $2,0 \mu \mathrm{M}$, cn raison de la forte homogénéité de la colonne d'eau. Ainsi, malgré des concentrations en nitrate relativement fortes en surface et une allure identique des courbes de vitesse du courant (figure $4 a$ ) et de flux d'eau (figure $6 b$ ) au cours des deux hivers, les flux en nitrate évoluent différemment entre les deux saisons puisqu'ils diminuent au cours de l'hiver 1992 et augmentent durant l'hiver 1993 (figure 6b).

La courbe des flux en NP à travers la radiale présente une allure très différente de celle des flux en nitrate (figure $6 b$ ) mais évolue de façon cohérente avec la courbe des flux en eau (figure $4 a$ ). Les flux en NP à travers la radiale sont principalement contrôlés par la vitesse et le flux en eau du courant. Pourtant, la variation du flux dans la couche profonde a un impact non négligeable sur la variabilité totale de la colonne d'eau (figure $6 b$ ) et est en relation avec l'exportation de matériel vers les couches profondes [7].

\subsection{Importance relative des apports hauturiers et rhodaniens}

Les sources d'enrichissements en sels nutritifs du golfe du Lion sont nombreuses (apports par la circulation générale et locale, apports fluviaux, processus de reminéralisation, fixation d'azote atmosphérique, précipitations, remise en suspension, diffusion et ruissellement) $[3,10$, $22,44]$. Dans cet ensemble, les deux sources principales sont constituées par la circulation générale et par le Rhône.

De récents bilans annuels des flux de matière du Rhône dans la zone du golfe du Lion fournissent une valeur des apports en azotc total de 80 à $100 \mathrm{kT} \mathrm{a}^{-1}[32,44]$. Cette quantité peut atteindre de 120 à $130 \mathrm{kT} \mathrm{a}^{-1}$ les années de fort débit [32]. Ces apports d'azote s'effcctuent principalement sous forme de nitrate $(80 \%)$, les formes organique dissoute et particulaire représentant 11 et $9 \%$ respectivement (tableau $I$ ). Afin de minimiser les erreurs relatives entre les apports du Rhône et de la circulation générale, nous utilisons ci-après la limite inférieure des intervalles précédemment donnés. En effet, la méthode de calcul adoptée dans notre étude constitue une sous-estimation du flux réel lié à la circulation générale.

Le flux en eau annuel moyen du Rhône est de $1700 \mathrm{~m}^{3}$ $s^{-1}$. Proportionnellement, le flux en eau moyen du CNM est mille fois supérieur $(1,1 \pm 0,2 \mathrm{~Sv}$ au cours des trois années du suivi). Pourtant, en termes de quantités de matière transportées les valeurs sont du même ordre de grandeur (figure 7). Cela s'explique par la relative richesse des eaux du Rhône [6] en comparaison des eaux oligotrophes ou mésotrophes du CNM $[2,7]$. Sur une base annuelle en terme d'azote total $(\mathrm{Nt})$, la quantité apportée par le Rhône représente environ $50 \%$ de la quantité de Nt traversant la radiale M1-M7 (figure $7 a$ ). Cela représente 20 à $30 \%$ de l'azote disponible dans le golfe du Lion (tableau $I$ ). Quant au nitrate, les apports du Rhône et de la circulation générale dans la couche 0-200 m sont comparables (figure 7a). Dans la couche $0-100 \mathrm{~m}$, les apports de nitrate liés à la circulation générale sont de $25 \pm 3 \mathrm{kT} \mathrm{a}^{-1}$, soit la moitié de la quantité introduite par le Rhône. En termes de NP, les flux apportés 


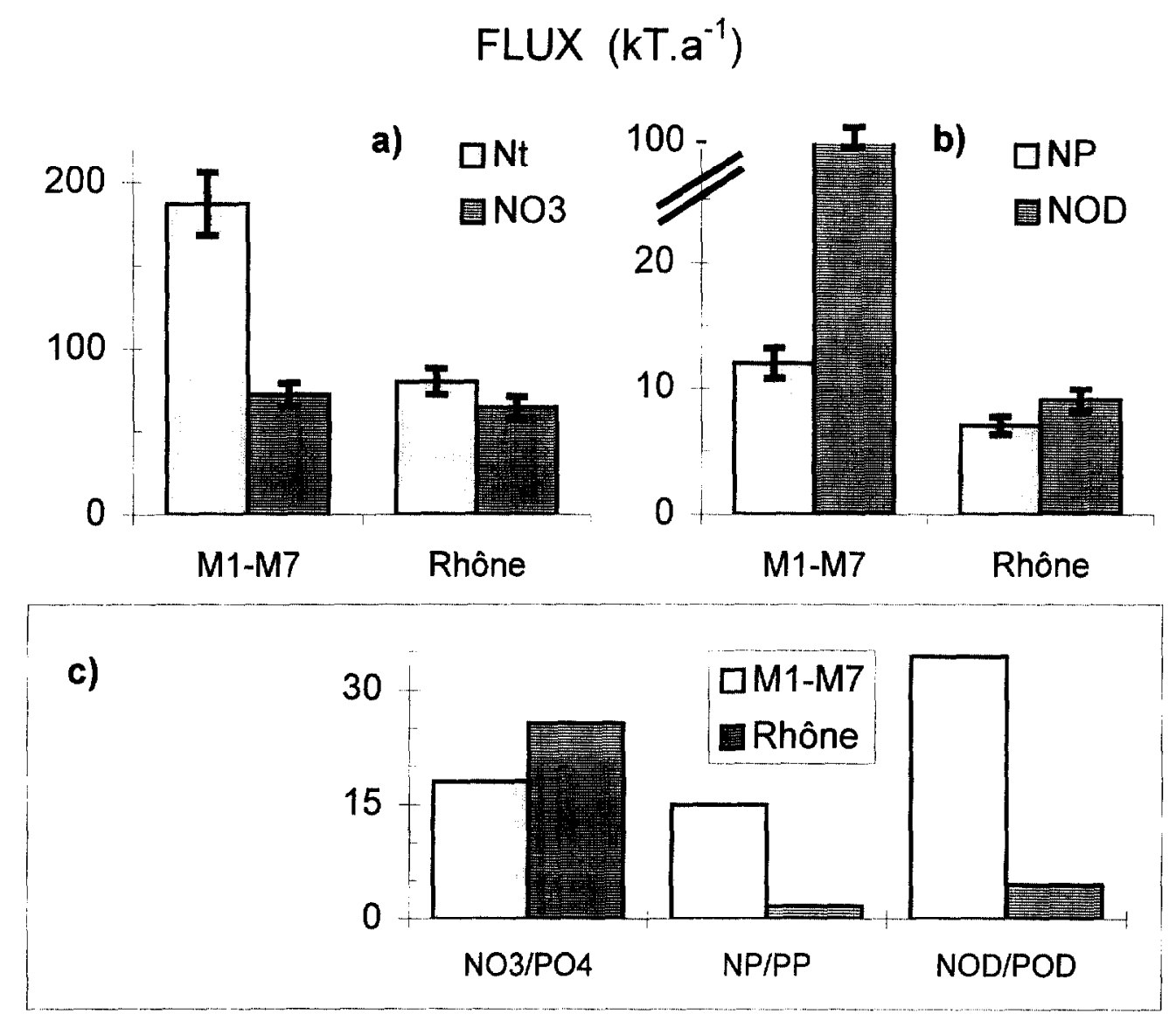

Figure 7. Apports annuels d'azote (en $\mathrm{kT} \mathrm{a}^{-1}$ ) dans le golfe du Lion par le Rhône et par la circulation générale à travers la radiale Ml-M7. a) Flux d'azote total (Nt) et de nitrate ( $\left.\mathrm{NO}_{3}\right)$. b) Flux d'azote particulaire (NP) et d'azote organique dissous (NOD). c) Valeurs du rapport azote sur phosphore dans les compartiments minéral, particulaire et orgarique dissous des apports totaux du Rhône et de la circulation générale.

Figure 7. Annual fluxes $\left(\mathrm{kT} \mathrm{a}^{-1}\right)$ introduced in the Gulf of Lions by the Rhone and by the general circulation across the M1-M7 transect. a) total nitrogen flux (Nt) and nitrate flux $\left(\mathrm{NO}_{3}\right)$, b) particulate nitrogen flux (NP) and dissolved organic nitrogen flux (NOD). c) Evolution of the nitrogen-phosphorus ratio in the various compartments: mineral, particulate and dissolved organic for the Rhone and general circulation inputs.

par ces deux sources sont équivalents, cela quelle que soit la couche considérée (figure $7 b$ ).

La principale différence entre les quantités d'azote introduites par le Rhône et par la circulation générale dans le golfe du Lion est à mettre en relation avec la forme organique dissoute (figure $7 b$ ). En effet, la quantité de NOD introduite par la circulation générale à travers la radiale M1-M7 est en moyenne sept à douze fois supérieure à la quantité apportée par le Rhône, selon la couche considérée (tableau I). Le NOD est particulièrement important en milicu marin (soit 40 à $90 \%$ de $\mathrm{Nt}$, [7]), mais ne représente qu'une forme minoritaire (moins de $10 \%$ ) dans les eaux du Rhône.

\subsection{Impact des apports de nitrate sur la productivité de la zone}

Les facteurs contrôlant l'impact des enrichissements en azote sur la production primaire et plus précisément des apports en nitrate sur la production nouvelle sont nombreux et leur action complexe et combinée. Il faut, en effet, considérer la quantité introduite et la localisation 
des apports (ponctuels dans le cas du Rhône, diffus dans le cas de la circulation générale). Il est également important de préciser la nature chimique sous laquelle cet azote est disponible pour le phytoplancton, ainsi que l'abondance relative de l'azote par rapport aux autres éléments nécessaires à la croissance du phytoplancton (phosphate). En effet, le rapport azote sur phosphore (N/P) des deux sources varie entre 15 et 25 dans la matière minérale, done proches de la valeur 16 du rapport de Redfield. Dans les compartiments particulaire et organique dissous, les apports rhodaniens sont excédentaircs en phosphore $(\mathrm{N} / \mathrm{P}<5)$, contrairement aux apports du large. La valeur dans les eaux du CNM pour NP/PP caractérise la matière vivante $(\sim 15)$, alors que la valeur pour le rapport NOD/ POD est supérieure à 30 et s'explique par les processus de reminéralisation plus rapides pour le phosphore que pour l'azote [37].

Parallèlement à l'étude du flux de matière transporté par la circulation générale, les variations mensuelles de la production primaire ont été suivies lors de chaque campagne à la mer, par des incubations in situ à l'aide de radioélément $\left({ }^{14} \mathrm{C}\right)$. Le résultat de ces mesures est détaillé dans Conan et al. [9]. Les apports annuels respectifs de nitrate du Rhône et de la circulation générale (tableau I) peuvent être convertis cn une quantité théorique de carbone, en considérant que l'ensemble du nitrate apporté est consommé sur place par le compartiment phytoplanctonique (rapport de consommation $\mathrm{C} / \mathrm{N}=6,6$ ). Cette quantité correspond à une estimation de la production nouvelle potentielle [28] dans la zone du golfe du Lion (tableau II). La quantité totale de nitrate injectée dans le golfe du Lion par le Rhône et par la circulation générale dans la couche superficielle $0-100 \mathrm{~m}$ à travers la radiale Ml-M7 pourrait être à l'origine d'une fixation biologique de 447 à $560 \mathrm{kT} \mathrm{a}^{-1}$, voire près de $900 \mathrm{kT} \mathrm{a}^{-1}$ dans les années de très forts débits rhodaniens. Dans cette fixation potentielle de carbone, les apports du Rhône et du large sont en moyenne de 70 à $80 \%$ et 20 à $40 \%$ respectivement.

En prenant une surface totale de $11000 \mathrm{~km}^{2}$ pour la zone du golfe du Lion, cette quantité de carbone correspond à une production nouvelle potentielle de 41 à $51 \mathrm{~g} \mathrm{~m}^{-2}$ a ${ }^{1}$ de carbone ou environ $80 \mathrm{~g} \mathrm{~m}^{2} \mathrm{a}^{-1}$ de carbone pour les années exceptionnelles. Cet intervalle est en accord avec les valeurs de production nouvelle mesurées ou déduites par méthode isotopique $\left({ }^{15} \mathrm{~N}\right)$, par bilan de consommation de sels nutritifs, par méthode ETS, par utilisation du facteur $f$ ou par mesures satellitales $[9,30$, 40]. Cet intervalle est cohérent avec celui de 50 à $56 \mathrm{~g} \mathrm{~m}^{-2} \mathrm{a}^{-1}$ de $\mathrm{C}$ obtenu à partir du bilan de la production primaire $\left({ }^{14} \mathrm{C}\right)$ et d'un facteur $\mathrm{f}$ de $0,3-0,4[9]$. Le résultat légèrement inférieur du bilan obtenu à partir de la conversion du flux de nitrate peut s'expliquer, d'une part, par la non-prise en compte de l'ensemble des sourecs de nitratc de la zonc puisque nous n'avons considéré que les apports rhodaniens et ceux du large et,

Tableau II. Estimation du bilan annuel de la production nouvelle potentielle de la zone du golfe du Lion (11 $\left.000 \mathrm{~km}^{2}\right)$ déduite des quantités de nitrate introduites par le Rhône et par la circulation générale dans la couche $0-100 \mathrm{~m}$ à travers $\mathrm{a}$ radiale Ml-M7. Les quantités de nitrate introduites sont extraites du tableau $I$ (min. = minimum, max. = maximum ; except. $=$ apport exceptionnel du Rhône, voir texte $)$, le coefficient de conversion molaire utilisé est $\mathrm{C} / \mathrm{N}=6,6$.

Table II. Estimation of the annual budget of the potential new production in the Gulf of Lions (area $=11000 \mathrm{~km}^{2}$ ) calculated from the nitrate inputs of the Rhone river and of the general circulation in the $0-100 \mathrm{~m}$ layer, across the M $1-\mathrm{M} 7$ transect. Total nitrate quantities are extracted from Table $I$ (min. = minimum, mlix. = maximum; except. = exceptional input by the river Rhone, cf. text) and the molar $\mathrm{C} / \mathrm{N}$ ratio use for conversion is taken as 6.6 .

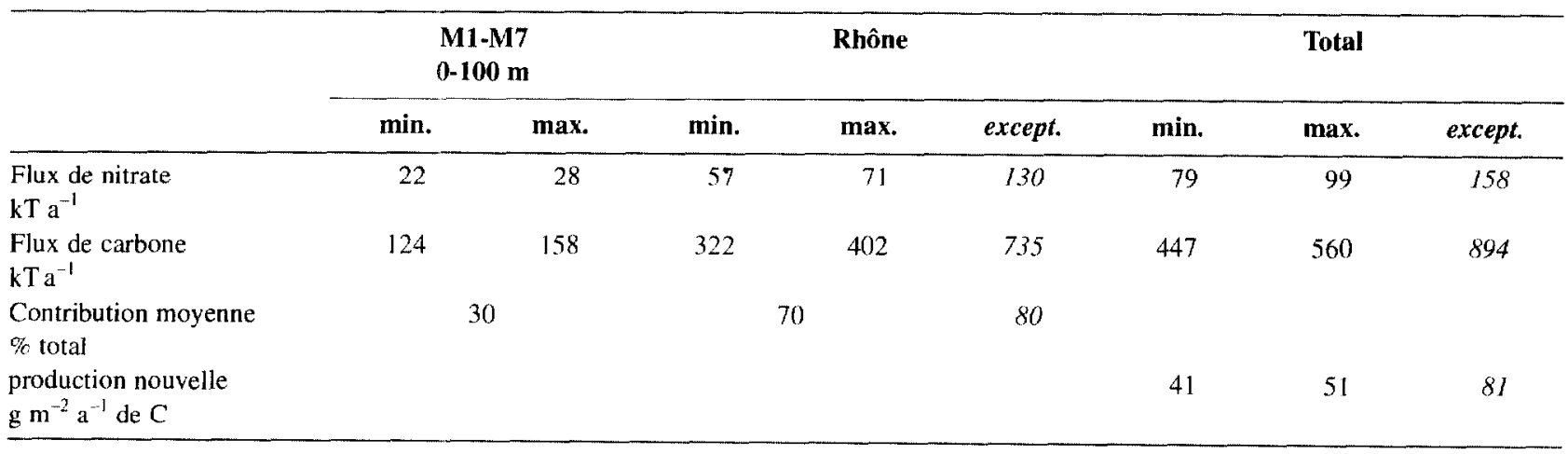


d'autre part, par la sous-estimation des flux réels du CNM comme déjà indiqué. Les deux erreurs cumulées introduites par ces deux approximations ne représenteraient alors pas plus de 10 à $30 \%$. Une autre source d'erreur est l'utilisation de différents facteurs (rapport C/ $\mathrm{N}$, facteur $f$ ) pour la conversion des mesures en grandeurs cherchées. Il reste que les méthodes utilisées fournissent des résultats relativement proches et qu'une valcur de la production nouvelle comprise entre 40 et $60 \mathrm{~g} \mathrm{~m}^{-2} \mathrm{a}^{-1}$ de carbone est une bonne estimation des potentialités d'exportation de la zone côtière du golfe du Lion.

\section{Remerciements}

Les auteurs remercient les équipages des navires océanographiques de la façade méditerranéenne pour leur professionnalisme au cours des nombreuses sorties effectuées dans le cadre de cette étude, ainsi que H. Massé, coordinateur du chantier Pnoc méditerranéen. Ils remercient également $D$. Lefèvre pour ses commentaires pendant la rédaction du manuscrit, ainsi que F. Cubizolles pour l'aide apportée dans la récolte des échantillons. Enfin, ils remercient les rapporteurs dont les commentaires ont permis d'améliorer la qualité de ce manuscrit.

\section{RÉFÉRENCES}

[1] Astraldi M., Gasparini J.P., Seasonal characteristics of the circulation in the western Mediterranean basin and their relationships with the atmospheric-climatic conditions, J. Geophys. Res. 97 (1992) 9531-9540.

[2] Berland B.R., Benzhitsky A.G., Burlakova Z.P., Georgieva L.V., Izmestiev A.M.A., Kholodov V.Y., Maestrini S.Y., Conditions hydrologiques estivales en Méditerranée, répartition du phytoplancton et de la matière organique, in : H.J. Minas et $P$. Nival (éd.). Océanographie pélagique méditerranéenne, Oceanol. Acta 9 (1988) 163-179.

[3] Béthoux J.P., Gentili B., The Mediterranean Sea, coastal and deep-sea signatures of climatic and environmental changes, $J$. Mar. Syst. 7 (1996) 383-394.

[4] Déthoux J.P., Prieur L., Hydrologie et circulation en Méditerranée nord-occidentale, Pétrole et techniques 299 (1983) 25 34.

[5] Byun S.K., Les eaux profondes de l'Atlantique nord-est. Hydrologie et circulation, thèse $3^{\mathrm{e}}$ cycle, univ. Bretagne occidentale (1980) $40 \mathrm{p}$.

[6] Cahet G., Fiala M., Jacques G., Panouse M., Production primaire au niveau de la thermocline en zone néritique de la Méditerranée nord-occidentale, Mar. Biol. 14 (1972) 32-40.

[7] Conan P., Variabilité et bilan de la production primaire en zone côtière (Méditerranée nord-occidentale, entrée du golfe du Lion) en relation avec les systèmes biologique, chimique et hydrodynamique (courant nord méditerranéen), thèse doct., univ. Méditerranée-Aix-Marseille II (1996) 290 p.

[8] Conan P., Millot C., Variability of the Northern Current in the Western Mediterranean Sea from February to June 1992 . Oceanol. Acta 18 (1995) 193-205.

[9] Conan P, Pujo-Pay M., Raimbault P., Leveau M., Variabilité hydrologique et biologique du golfe du Lion. Il. Productivité sur le bord interne du courant, Oceanol. Acta 21 (6) (1998) $767-782$.
[10] Coste B., Rôle des apports nutritifs minéraux rhodaniens sur la production organique des eaux du golfe du Lion, Téthys 6 (1974) 727-740.

[11] Crépon M., Boukthir M., Effect of deep water formation of the Ligurian bassin, Annal. Geophys. 5 (1987) 43-48.

[12] D'Heilly C., Millot C., Monaco A., Got H., Hydrodynamic study of the Petit Rhône canyon, Deep-Sea Res. 35 (1988) $465-471$.

[13] Durrieu de Madron X.. Transport de la matière particulaire en suspension sur les marges continentales du golfe du Lion (Méditerranée occidentale), du bassin des Sporades (Méditerranée orientale) et du golfe de Gascogne (Atlantique nord-est), thèse doct., univ. Aix-Marseille-II, Neuchatel (1991) 59 p.

[14] Durrieu de Madron X., Panouse M., Transport de matière particulaire en suspension sur le plateau continental du golfe du Lion. Situation estivale et hivernale, C. R. Acad. Sci. 322 (1996) 106I-1070.

[15] Fieux M., Contribution à l'étude hydrologique et dynamique du golfe du Lion en période hivernale, thèse $3^{2}$ cycle, univ. Paris-VI (1973) $90 \mathrm{p}$.

[16] Fieux M., Formation d'eau dense sur le plateau continental du golfe du Lion, in : La formation des eaux océaniques profondes, Coll. Int. CNRS 215 (1974) 165-189.

[17] Font J., The path of Levantine Intermediate Water to the Alboran Sca, Deep-Sea Res. 34 (1987) 1745-1755.

[18] Font J., Garcia-Ladona E., Gorriz E.G., The seasonality of mesoscale motion in the Northern Current of the western Mediterranean: several years of evidence, Oceanol. Acta 18 (1995) 207-221.

[19] Gostan J., Contribution à ]'étude hydrologique du bassin liguro-provençal entre la Riviera et la Corse, thèse doct., univ. Paris (1968) $206 \mathrm{p}$.

[20] Groupe Médoc, La formation des eaux océaniques profondes, Coll. inter. CNRS 215 (1972) $278 \mathrm{p}$. 
[21] Holm-Hansen O., Riemann B., Chlorophyll a determination: improvements in methodology, Oikos 30 (1978) 438-447.

[22] Jacques G., Minas H.J., Minas M., Nival P., Influence des conditions hivernales sur les productions phyto et zooplanctoniques en Méditerranée nord-occidentale, II. Biomasse et production planctonique, Mar. Biol. 23 (1973) 251-265.

[23] K]ein P., Coste B., Effects of wind stress variability on nutrient transport into the mixed layer, Deep-Sea Res. 31 (1984) 21 27.

[24] Mann K. H., Lazier J.R.N., Dynamics of marine ecosystems / Biological-physical interactions in the oceans, Blackwell Sci Pub., Londres, 1991, $466 \mathrm{p}$.

[25] Millot C., The Gulf of Lion's hydrodynamics, Cont. Shelf Res. 10 (1990) 885-894.

[26] Millot C., Are there major differences between the largest mediterranean seas? A preliminary investigation, Bull. Inst. Océanogr. 11 (1992) 3-25.

[27] Millot C., Wald L., The effects of Mistral wind on the Ligurian current near Provence, Oceanol. Acta 4 (1980) 399-402.

[28] Minas H.J., Recherches sur la production organique primaire dans le bassin méditerranéen nord-occidental, Rapports avec les phénomènes hydrologiques, thèse doct., univ. Aix-Marseille-II (1968) $228 \mathrm{p}$.

[29] Monaco A., Biscay P., Soyer S., Pocklington R., Heussner S., Particles fluxes and ecosystem response on a continental margin: the 1985-1988 Mediterranean Ecomarge experiment. Cont. Shelf Res. 10 (1990) 809-839.

[30] Morel A., André J.M., Pigment distribution and primary production in the Western Mediterranean as derived and modeled from coastal zone scanner observation, J. Geophys. Res. 96 (1991) 12,685-12,698.

[31] Moskalenko L.V., Ovchinnikov [.M., Geostrophic circulation in the Mediterranean Sea, Oceanology 31 (1991) 668-674.

[32] Moutin T., Raimbault P., Golterman H.L., Coste B., The input of nutrients by the Rhône river into the Mediterranean Sea: recent observations and comparison with earlier data. Hydrobio (1998) (sous presse).

[33] Naudin J.J., Cauwet G., Leveau M., Lochet F., Pauc H., Romano J.C., Sempere R., Le néphéloide benthique au large du Rhône. Transferts particulaires à l'interface continentocéan, Oceanol. Acta 16 (1992) 621-638.

[34] Naudin J.J., Cauwet G., Fajon C., Oriol L., Terzic S., Devenon J.L., Broche P., Experimental study of the Rhône river plume. Part II: Biological processes, Oceanol. Acta, submitted.
[35] Prieur L., Systèmes frontaux en mer ligure à partir des mesures multiparamétriques en continu, Courrier Mediprod, Lab. Arago Banyuls 2 (1985) 2-5.

[36] Pujo-Pay M., Conan P., Raimbault P., Particulate and dissolved organic nitrogen and phosphorus in the northwestern Mediterranean Sea (Eros 2000-Discovery cruise 1993), Wat. Poll. Res. Rep. EROS 2000, $5^{\text {}}$ workshop 28-30 mars 1994, Martin J.M., Barth H. (éd.) (1994) 79-86

[37] Pujo-Pay M., Conan P., Variability and export of Dissolved Organic Nitrogen (DON) in the North Western Mediterranean Sea, Deep-Sea Res. (en révision).

[38] Pujo-Pay M., Raimbault P., Improvement of the wet-oxidation procedure for similtaneous determination of particulate organic nitrogen and phosphorus collected on filters, Mar. Ecol. Prog. Ser. 105 (1994) 203-207.

[39] Sammari S., Millot C., Prieur L., Aspects of the seasonal and mesoscale variabilities of the Northern Current in the western Mediterranean Sea inferred from PROLIG-2 and PROS-6 experiments, Deep-Sea Res. 42 (1995) 893-917.

[40] Sournia A., La production primaire planctonique en Méditerranée -- Essai de mise à jour -, Newsletter of cooperative investigations in the Mediterranean, Monaco 5 (1973) $127 \mathrm{p}$.

[41] Taupier-Letage I., Millot C., Conan P., Dickey T., Mano D., Foley D., Temporal variability of biological parameters in the North-western Mediterranean, Rapp. Comm. Int. Mer Médit. 34 (1995) 217

[42] Travers A., Travers M., Données sur quelques facteurs de l'écologie du plancton dans la région de Marseille. 2. La température, les précipitations, la stabilité et la circulation des eaux, Téthys 4 (1972) 515-534.

[43] Tréguer P., Le Corre P., Manuel d'analyses des sels nutritifs dans l'eau de mer, laboratoire d'océanographie chimique, univ. Bretagne occidentale, Brest (1975) $110 \mathrm{p}$.

[44] Tusseau M.H., Mouchel J.M.. Nitrogen inputs to the gulf of Lions via the Rhone river. Wat. Poll. Res. Rep. EROS 2000, $5^{\circ}$ workshop 28-30 mars 1994, J. M. Martin et H. Barth (éd.) (1994) 49-60.

[45] Wood E.P.K., Armstrong F.A.J., Richards F.A., Determination of nitrate in seawater by cadmium cooper reduction to nitrite, J. Mar. Biol. Assoc. U.K. 47 (1967) 23-31.

[46] Wond J.D., Wave-induced shear instability in summer thermocline, J. Fluid. Mech. 32 (1968) 79l-800. 